\title{
Efficacy analysis of Arbidol treatment in patients with 2019 novel coronavirus pneumonia: a retrospective cohort study
}

\author{
Nanjin Chen ${ }^{1 *}$, Xiaodan Wang ${ }^{1 *}$, Sheng Zhang ${ }^{2}$, Ronghai Lin ${ }^{3}$, Yongpo Jiang ${ }^{2 \wedge}$ \\ ${ }^{1}$ Department of Anesthesiology, Taizhou Hospital of Zhejiang Province Affiliated to Wenzhou Medical University, Taizhou, China; ${ }^{2}$ Department of \\ Critical Care Medicine, Taizhou Hospital of Zhejiang Province Affiliated to Wenzhou Medical University, Taizhou, China; ${ }^{3}$ Department of Critical \\ Care Medicine, Taizhou Municipal Hospital, Taizhou, China \\ Contributions: (I) Conception and design: J Jiang; (II) Administrative support: S Zhang; (III) Provision of study materials or patients: J Jiang; (IV) \\ Collection and assembly of data: N Chen; (V) Data analysis and interpretation: N Chen; (VI) Manuscript writing: All authors; (VII) Final approval of \\ manuscript: All authors. \\ \#These authors contributed equally to this work. \\ Correspondence to: Yongpo Jiang. Department of Critical Care Medicine, Taizhou Hospital of Zhejiang Province Affiliated to Wenzhou Medical \\ University, Taizhou 317000, China. Email: 7719@enzemed.com.
}

\begin{abstract}
Background: The aim of this study was to determine whether Arbidol has a good antiviral effect on coronavirus disease 2019 (COVID-19).

Methods: A retrospective cohort study was performed in one of the treatment centers for COVID-19 patients in China from January 2020 to March 2020. The antiviral drug Arbidol (ARB) was administrated to some of the patients at $0.2 \mathrm{~g}$ tid po for 7 to 10 days. According to whether patients were given ARB, they were divided into 2 groups: the ARB group and the Non-ARB group. The primary outcome was the 14-day COVID-19 negativity rate.

Results: Of 146 patients, 140 were included. A total of 79 (56.4\%) patients received ARB during hospitalization. In the overall cohort, the time of COVID-19 negativity in the ARB group compared with the Non-ARB group was 12.9 days versus 12.7 days $(\mathrm{P}=0.175 ;>0.05)$. The rates of 14 -day COVID-19 negativity were $60.8 \%$ and $65.6 \%$ in the $\mathrm{ARB}$ and non-ARB groups, respectively $(\mathrm{P}=0.559 ;>0.05)$. Using an adjusted model, there were no obvious differences in the time of COVID-19 negativity and the rates of 14-day COVID-19 negativity ( $\mathrm{P}>0.05)$. According to Kaplan-Meier analysis, the probabilities of 14-day COVID-19 negativity were similar in the 2 groups ( $\log$-rank $\mathrm{P}=0.130 ;>0.05)$. In a multivariate Cox analysis, the variables of age [hazard ratio (HR) 0.91, 95\% confidence interval (CI): 0.83 to $0.99 ; \mathrm{P}=0.039$ ] and glucose (HR 0.90, 95\% CI: 0.82 to $0.98 ; \mathrm{P}=0.021$ ) were independently associated with 14 -day COVID-19 negativity.
\end{abstract}

Conclusions: Our results suggest that there was no apparent favorable clinical response with ARB both in clinical symptoms and the 14-day COVID-19 negativity rate.

Keywords: Coronavirus disease 2019 (COVID-19); COVID-19-related pneumonia; Arbidol; antiviral treatment; negativity rate

Submitted Aug 06, 2021. Accepted for publication Oct 09, 2021.

doi: 10.21037/apm-21-2397

View this article at: https://dx.doi.org/10.21037/apm-21-2397

^ ORCID: 0000-0002-5681-3663. 


\section{Introduction}

Coronavirus disease 2019 (COVID-19) is caused by a novel beta-coronavirus recently named severe acute respiratory syndrome coronavirus-2 (SARS-CoV-2) (1-3). The 2019 novel coronavirus-infected pneumonia (NCIP) has become a worldwide pandemic that is overwhelming health care systems globally $(4,5)$. The most severe symptom of COVID-19 is similar to severe acute respiratory syndrome (SARS) (1). The person-to-person transmission route of COVID-19 is similar to that of SARS (6). Supportive care and antiviral therapy remain the mainstay for treating patients with COVID-19; however, there is currently no proven effective specific antiviral drug available (7). Therefore, the antiviral activity drugs against SARS-CoV-2 are urgently needed to treat COVID-19 patients.

Arbidol (ARB) (also known as umifenovir) is a nonnucleoside antiviral agent approved in China for the prevention and treatment of influenza and other respiratory viruses. It is a broad-spectrum antiviral drug that has proven antiviral effects against influenza, Lassa, and Ebola, among others $(8,9)$. The antiviral effect of ARB not only affects hemagglutinin but also can inhibit the endocytosis and fusion of the virus on the surface of the host cell. By blocking the virus outside of the host cell, ARB prevents the virus from host cell entry. It has been found that ARB has a good inhibitory effect against SARS-CoV-2 in vitro (10). Some academics have recommended to use of ARB in patients with COVID-19 (7,11-13); however, the antiviral effect of $\mathrm{ARB}$ on COVID-19-related pneumonia is still controversial $(10,14)$. We retrospectively collected clinical data, including ARB treatment records and the time of COVID-19 negativity. This study might provide information on the clinical application of ARB in the treatment of SARS-CoV-2 infection. We present the following article in accordance with the STROBE reporting checklist (available at https:// dx.doi.org/10.21037/apm-21-2397).

\section{Methods}

\section{Study design and patients}

This study was a retrospective, observational, single-center study. Ethics approval was granted by the Enze Hospital Ethics Committee of Taizhou Enze Medical Center (Group) (Also called Taizhou Hospital of Zhejiang Province) (K20200204). All patients from Enze Hospital, Taizhou Enze Medical Center (Group), from 31 January 2020, to 11 May 2020 were included in the analysis. The participants were divided into 2 groups according to whether they received ARB: the ARB group, in which ARB was administered, and the Non-ARB group, in which ARB was not administered. The dosage and period of administration of ARB for COVID-19 treatment were $0.2 \mathrm{~g}$ tid po for 7-10 days. The primary clinical outcome was the time after the initiation of antiviral therapy at which the nucleic acid test for SARS-CoV-2 became negative. The secondary outcomes were the duration of fever and symptoms. The study was conducted in accordance with the Declaration of Helsinki (as revised in 2013). Written informed consent was provided by all participants before inclusion.

PCR-confirmed cases of COVID-19-infected pneumonia were consecutively included. The diagnostic criteria were as follows: (I) reverse transcription-polymerase chain reaction (RT-PCR)-confirmed infection with COVID-19; and (II) lung involvement confirmed with chest imaging. The diagnostic criteria for severe patients were as follows: any of the following: respiratory rate $(\mathrm{RR})>30$ breaths/min at rest, mean oxygen saturation $\leq 93 \%$; arterial oxygen pressure/ oxygen concentration $\left(\mathrm{PaO}_{2} / \mathrm{FiO}_{2}\right) \leq 300 \mathrm{mmHg}$ (15). The exclusion criteria were as follows: pregnancy; age $<18$ years; and incomplete data.

\section{Data collection}

All participant data were extracted from electronic medical records, which included epidemiological characteristics, clinical signs and symptoms, and laboratory findings [including interleukin (IL)-2, IL-4, IL-6, IL-10, tumor necrosis factor (TNF), interferon (IFN)- $\gamma$, and COVID-19 RNA]. Chest computed tomography (CT) was employed as the imaging study. All participants included in the analysis were patients from Enze Hospital, Taizhou Enze Medical Center (Group).

\section{Statistical analysis}

Data were expressed as the means \pm standard deviation (SD), medians with ranges, or percentages with numbers of patients. Normally distributed data was evaluated by using the Shapiro-Wilk test $(\mathrm{P}>0.05)$. Continuous variables that were normally distributed were carried out using Student's $t$-test or the corrected $t$-test. Non-parametric distribution variables were performed using the Mann-Whitney $U$ test. Comparisons of categorical variables were performed using the $\chi^{2}$ test or Fisher's test. Kaplan-Meier survival curves and the log-rank test were performed to estimate the 


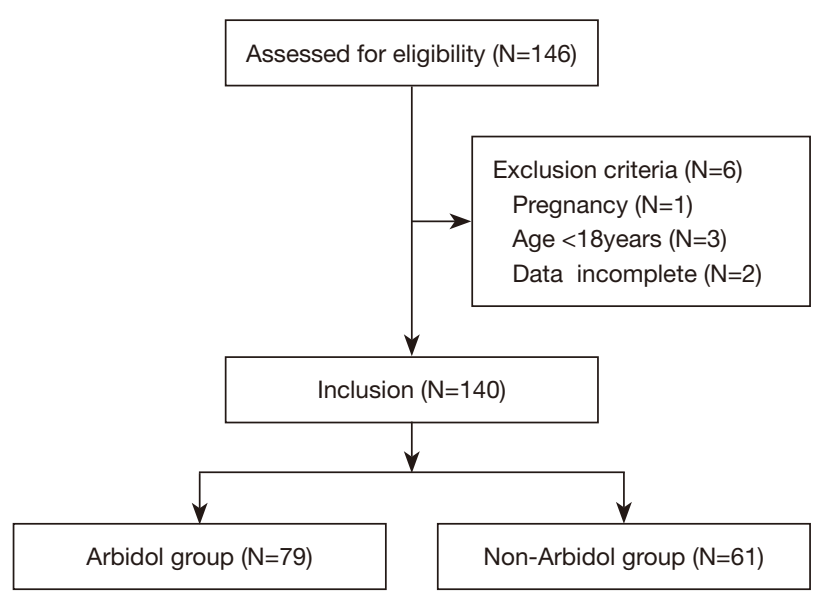

Figure 1 Flow chart of the enrollment of this study.

14-day COVID-19 negativity rate. The prognostic value of the variables was assessed using a univariate Cox proportional hazards regression adjusted model. Statistical significance was considered when $\mathrm{P}<0.05$. All statistical analyses were performed with SPSS 26.0 (IBM Corp., Armonk, NY, USA) and GraphPad Prism 8.0 (GraphPad Software, San Diego, CA, USA).

\section{Results}

\section{Participant and baseline characteristics}

Of 146 patients, 140 patients with COVID-19 were included based on the inclusion and exclusion criteria (Figure 1). Among the 140 participants, 79 (56.4\%) were treated with ARB during hospitalization. The mean age was 48 years, $53.6 \%$ were males, and $72.9 \%$ had a fever. In addition, $25 \%$ [35] of participants were identified as having severe NICP and none of the severe patients progressed to critical illness or died (Table 1). The participants in the two groups had normal white blood cell counts, and most participants had obvious lymphopenia. The participants in the ARB group had no prominent laboratory abnormalities (i.e., routine blood test, biochemical blood tests, hemagglutination series, blood gas analysis) compared with the non-ARB participants. The median time from symptom onset to hospitalization was 2 days (range, 0 to 14 days). The median time from symptom onset to the initiation of antiviral therapy was 4.0 days (range, 0 to 20 days) (Table S1). The baseline oxygen support of the participants between the 2 groups was not significantly different (Table S1). No significant differences were found between the 2 groups in the levels of inflammatory factors, such as IL-2, IL-4, IL-6, IL-10, TNF- $\alpha$, and TFN- $\gamma$ (Figure S1).

\section{Primary and secondary outcomes}

The mean time after the initiation of antiviral therapy at which the nucleic acid tests for SARS-CoV-2 became negative was $12.8 \pm 7.1$ days and the 14-day COVID-19 negativity rate was $62.9 \%$ in the overall cohort (Table 2). The probabilities of negativity for COVID-19 at 14 days were $60.8 \%$ and $65.6 \%$ in the ARB group and non-ARB group, respectively, and there was no significant difference between the 2 groups $(\mathrm{P}=0.559)$ (Table 2). The duration of fever was $5.8 \pm 2.1$ and $5.5 \pm 2.2$ days, respectively, and there was no significant difference between the 2 groups $(\mathrm{P}=0.337)$. The duration of symptoms was $10.2 \pm 5.0$ and $10.6 \pm 5.1$ days, respectively, and there was no significant difference between the 2 groups $(\mathrm{P}=0.670)$ (Table 2). There were no obvious differences in the time of COVID-19 negativity, rates of 14-day COVID-19 negativity, duration of fever, and the duration of symptoms using the adjusted model $(\mathrm{P}>0.05)$.

\section{Kaplan-Meier survival analysis}

Kaplan-Meier survival analysis was used to compare the rate of 14-day COVID-19 negativity between the 2 groups. The probability of COVID-19 negativity at 14 days was similar in the ARB group and non-ARB group ( $\log$-rank $\mathrm{P}=0.130 ;>0.05$ ) (Figure $2 A$ ). In a subgroup analysis of participants according to severity, in the non-severe population, the probability of COVID-19 negativity at 14 days was not significantly different between the ARB group and the non-ARB group $(\mathrm{P}=0.06 ;>0.05)$ (Figure $2 B)$. In the severe population, the probability of COVID-19 negativity at 14 days was also not significantly different between the ARB group and the nonARB group $(\mathrm{P}=0.655 ;>0.05)$ (Figure $2 C$ ).

\section{Cox regression analysis}

The univariate Cox regression analysis showed that age, use of gamma globulin, use of glucocorticoid, absolute lymphocyte value, creatine kinase, and $\mathrm{PaO}_{2} / \mathrm{FiO}_{2}$ were significantly $(\mathrm{P}<0.05)$ associated with 14 -day COVID-19 negativity. In the multivariate analysis, the variables of age [hazard ratio (HR) $0.91,95 \%$ confidence interval (CI): 0.83 to $0.99 ; \mathrm{P}=0.039$ ] and glucose (HR $0.90,95 \% \mathrm{CI}: 0.82$ to $0.98 ; \mathrm{P}=0.021$ ) were independently associated with 14 -day 
Table 1 Baseline characteristics of 140 patients with COVID-19

\begin{tabular}{|c|c|c|c|c|}
\hline Characteristic & All patients $(\mathrm{N}=140)$ & Non-ARB group $(\mathrm{N}=61)$ & ARB group $(\mathrm{N}=79)$ & $P^{a}$ \\
\hline Gender (male), n (\%) & $75(53.6)$ & $39(63.9)$ & $36(45.6)$ & 0.031 \\
\hline Severe patients, n (\%) & $35(25.0)$ & $13(21.3)$ & $22(27.8$ & 0.376 \\
\hline Smoking history, n (\%) & $13(9.2)$ & $7(11.3)$ & $6(7.6)$ & 0.433 \\
\hline \multicolumn{5}{|l|}{ Exposure history, n (\%) } \\
\hline Recently been to Wuhan & $67(47.9)$ & $25(41.0)$ & $42(53.2)$ & 0.113 \\
\hline Contact with people from Wuhan & $72(51.4)$ & $35(57.4)$ & $37(46.8)$ & 0.190 \\
\hline \multicolumn{5}{|l|}{ Comorbidities, n (\%) } \\
\hline Hypertension & $23(16.4)$ & $6(9.8)$ & $17(21.5)$ & 0.064 \\
\hline Chronic liver disease & $4(2.9)$ & $3(4.9)$ & $1(1.3)$ & 0.318 \\
\hline \multicolumn{5}{|l|}{ Symptoms } \\
\hline Fever, n (\%) & $102(72.9)$ & $42(68.9)$ & $60(75.9)$ & 0.349 \\
\hline Highest temperature, ${ }^{\circ} \mathrm{C}$ & $38.3 \pm 0.6$ & $38.3 \pm 0.7$ & $38.2 \pm 0.5$ & 0.745 \\
\hline Cough, n (\%) & $90(64.3)$ & $41(67.2)$ & $49(62.0)$ & 0.525 \\
\hline Sore throat, n (\%) & $15(10.7)$ & $4(6.6)$ & $11(13.9)$ & 0.162 \\
\hline Headache, n (\%) & $12(8.6)$ & $3(4.9)$ & $9(11.4)$ & 0.175 \\
\hline Diarrhea, n (\%) & $13(9.3)$ & $4(6.6)$ & $9(11.4)$ & 0.328 \\
\hline
\end{tabular}

a, Student's $t$-test, Mann-Whitney $U$ test, $\chi^{2}$ test and Fisher test. COVID-19, coronavirus disease 2019; ARB, Arbidol; SD, standard deviation.

Table 2 Clinical outcomes

\begin{tabular}{|c|c|c|c|c|c|}
\hline Outcomes & All patients $(\mathrm{N}=140)$ & Non-ARB group $(\mathrm{N}=61)$ & ARB Group $(\mathrm{N}=79)$ & $P^{a}$ & $\mathrm{P}^{\mathrm{b}}$ \\
\hline Days until virus negativity, mean $\pm S D$, days & $12.8 \pm 7.1$ & $12.9 \pm 9.2$ & $12.7 \pm 5.1$ & 0.175 & 0.055 \\
\hline $14-$ day virus negativity rate, $\mathrm{n}(\%)$ & 88 (62.9) & $40(65.6)$ & $48(60.8)$ & 0.559 & 0.322 \\
\hline \multicolumn{6}{|l|}{ Secondary outcomes } \\
\hline Duration of symptoms, mean $\pm S D$, days & $10.4 \pm 5.0$ & $10.2 \pm 5.0$ & $10.6 \pm 5.1$ & 0.670 & 0.792 \\
\hline
\end{tabular}

\footnotetext{
${ }^{\mathrm{a}}$, non-adjusted model adjusted for: none; ${ }^{\mathrm{b}}$, adjusted for: gender. ARB, Arbidol; SD, standard deviation.
} 

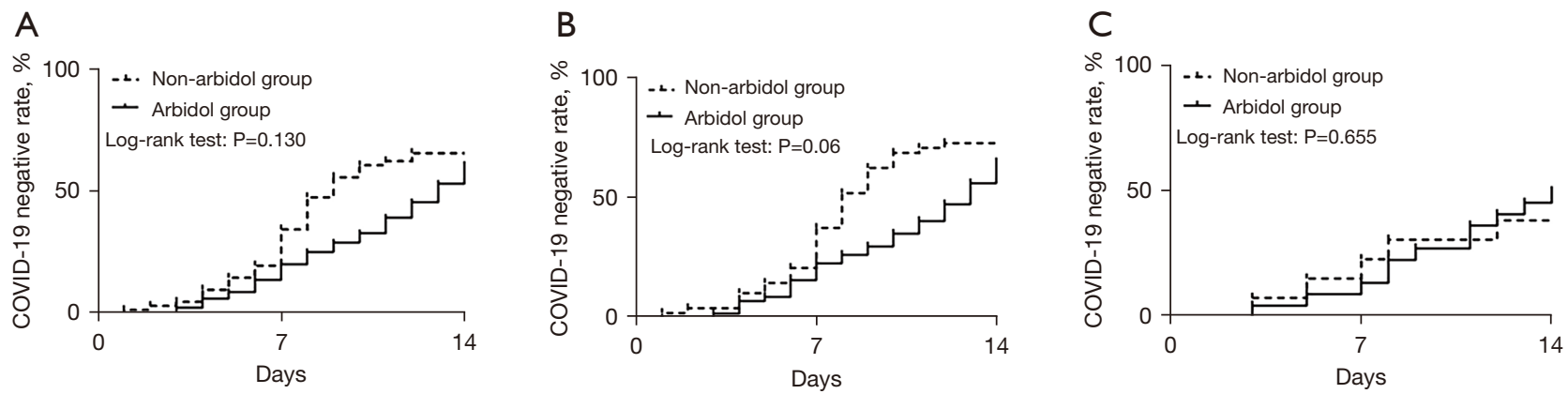

Figure 2 Kaplan-Meier curves stratified based on the time to SARS-CoV-2 negativity. (A) In the overall cohort, the probabilities of 14-day COVID-19 negativity were similar in the 2 groups $(\mathrm{P}=0.130 ;>0.05)$. (B) In a subgroup analysis of non-severe patients, no significant differences were found between the ARB group and Non-ARB group in the 14-day COVID-19 negativity rate (P=0.06; $>0.05)$. (C) In a subgroup analysis of severe patients, no significant differences were found between the ARB group and Non-ARB group in the 14-day COVID-19 negativity rate $(\mathrm{P}=0.655 ;>0.05)$. $\mathrm{P}$ values were for differences in the time to SARS-CoV-2 negativity as assessed by the log-rank test. SARS-CoV-2, severe acute respiratory syndrome coronavirus-2; COVID-19, coronavirus disease 2019; ARB, Arbidol.

Table 3 Univariate and multivariate analyses for 14-day COVID-19 negativity

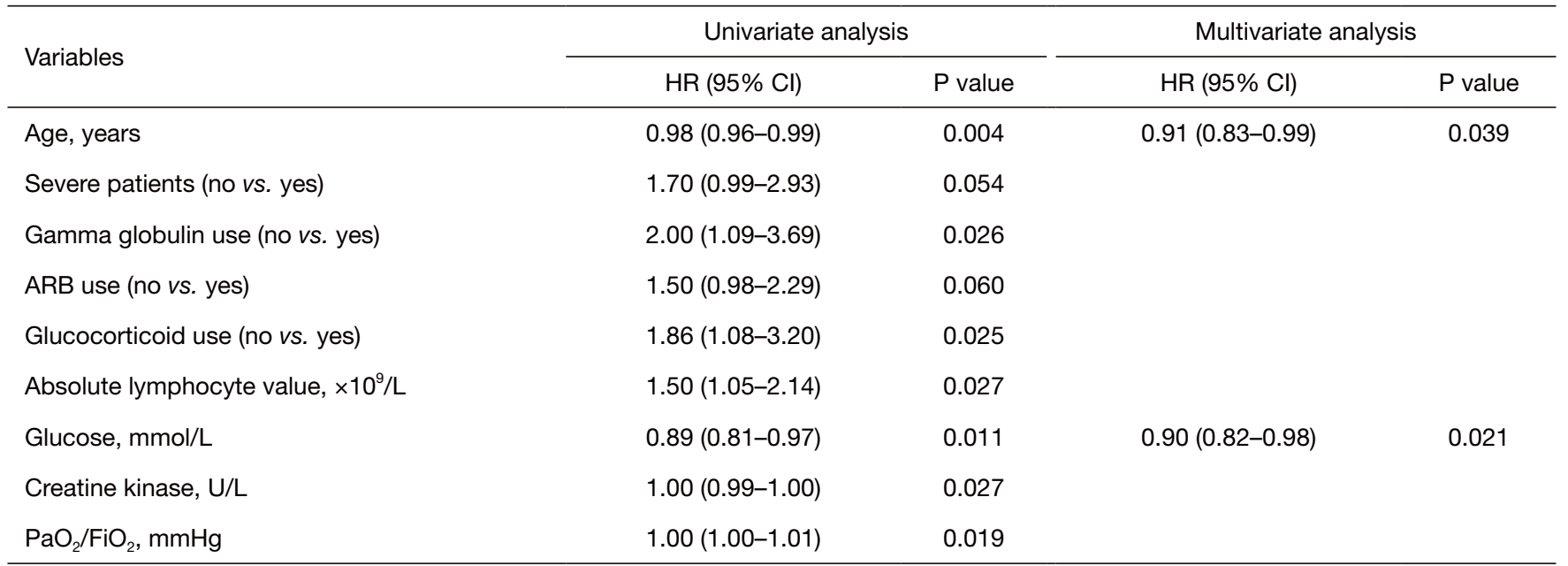

Adjusted for: gender. COVID-19, coronavirus disease 2019; ARB, Arbidol; HR, hazard ratio; $\mathrm{Cl}$, confidence interval; $\mathrm{PaO}_{2} / \mathrm{FiO}_{2}$, arterial oxygen pressure/oxygen concentration.

COVID-19 negativity (Table 3).

\section{Discussion}

The highly infectious SARS-CoV-2 is a single-stranded RNA beta-coronavirus (2). Due to the evidence of humanto-human transmission, early isolation and early antiviral treatment are very important for patients with COVID-19 $(16,17)$. Currently, several antiviral agents have been suggested as treatment options for COVID-19, including remdesivir, chloroquine, and lopinavir-ritonavir, but no agent has yet been shown to have clinical benefits in patients with
COVID-19 (18-20). The surface structural spike glycoprotein in coronaviruses is one of the most important therapeutic targets for antiviral agents because of its important role in virus-cell receptor interactions $(21,22)$. Fortunately, some scientists have found that ARB has a certain inhibitory effect on SARS-CoV-2 in vitro, and ARB is currently the only known inhibitor of hemagglutinin (the spike-like glycoprotein on the envelope of the virus) $(8,10,23)$. In a clinical setting, a study reported that among 4 patients who were given antiviral treatments, including lopinavir/ritonavir (Kaletra $\left.{ }^{\circledR}\right)$ and ARB; 2 patients were confirmed to be SARS-CoV-2negative and were discharged (11). In a retrospective cohort 
study on 16 patients who received oral ARB combined with lopinavir/ritonavir, there was an apparent favorable clinical response (24). However, another study suggested that ARB might not improve the prognosis or accelerate SARS-CoV2-negativity in non-intensive care unit (ICU) patients (14). In this study, we found that the time to negativity for the virus in patients with COVID-19 who were given SRB was not shorter than that in Non-ARB-treated patients. The rates of 14 -day COVID-19 negativity were $60.8 \%$ and $65.6 \%$ in the $\mathrm{ARB}$ and non-ARB groups, respectively $(\mathrm{P}=0.559 ;>0.05)$ (Table 2). The improvement in clinical symptoms was not significantly different between the 2 groups. As we know, there are large differences in the antiviral effects of in vitro and in vivo drugs. A study of the concentrations of agents in vivo is also necessary. In this study, we found that $\mathrm{ARB}$ does not have an antiviral effect in patients with COVID-19. However, a large-sample randomized controlled trial (RCT) is needed to assess the efficacy of ARB.

The factors affecting the time of COVID-19 negativity are unknown, but they are vital for the treatment time. One study reported that serum lactate dehydrogenase or creatine kinase decline may predict a favorable response to treatment of COVID-19 infection (25). In this study, we found that age, the use of gamma globulin and glucocorticoid, glucose, creatine kinase, and $\mathrm{PaO} 2 / \mathrm{FiO} 2$ were associated with 14-day COVID-19 negativity in the univariate analysis. In the multivariate analysis, the variables of age (HR 0.91 , 95\% CI: 0.83 to $0.99 ; \mathrm{P}=0.039$ ) and glucose (HR 0.90, 95\% CI: 0.82 to $0.98 ; \mathrm{P}=0.021$ ) were independently associated with 14-day COVID-19 negativity (Table 3). This study was limited by the small size of the retrospective cohort. Furthermore, our study did not collect viral load data to confirm the antiviral effects of ARB or if there was any association between the baseline viral load and viral suppression, and clinical response.

\section{Conclusions}

In summary, our study suggests that Arbidol might not improve the prognosis or accelerate SARS-CoV-2 clearance. Further RCTs may be needed to evaluate the antiviral effect of ARB against SARS-CoV-2. It is necessary to find new potential targets for antiviral drugs to control the COVID-19 pandemic.

\section{Acknowledgments}

Acknowledgments to all physicians and nurses in Taizhou
Public Health Center.

Funding: This work was supported by The Science and Technology Project of Taizhou (21ywb05) and the Natural Science Foundation of Zhejiang Province (Y20H150029).

\section{Footnote}

Reporting Checklist: The authors have completed the STROBE reporting checklist. Available at https://dx.doi. org/10.21037/apm-21-2397

Data Sharing Statement: Available at https://dx.doi. org/10.21037/apm-21-2397

Conflicts of Interest: All authors have completed the ICMJE uniform disclosure form (available at https://dx.doi. org/10.21037/apm-21-2397). The authors have no conflicts of interest to declare.

Ethical Statement: The authors are accountable for all aspects of the work in ensuring that questions related to the accuracy or integrity of any part of the work are appropriately investigated and resolved. The study was conducted in accordance with the Declaration of Helsinki (as revised in 2013). Ethics approval was granted by Hospital Ethics Committee of Enze Hospital of Taizhou Enze Medical Center (Group) (Also called Taizhou Hospital of Zhejiang Province) (K20200204). Written informed consent was provided by all participants before inclusion.

Open Access Statement: This is an Open Access article distributed in accordance with the Creative Commons Attribution-NonCommercial-NoDerivs 4.0 International License (CC BY-NC-ND 4.0), which permits the noncommercial replication and distribution of the article with the strict proviso that no changes or edits are made and the original work is properly cited (including links to both the formal publication through the relevant DOI and the license). See: https://creativecommons.org/licenses/by-nc-nd/4.0/.

\section{References}

1. Wang D, Hu B, Hu C, et al. Clinical Characteristics of 138 Hospitalized Patients With 2019 Novel CoronavirusInfected Pneumonia in Wuhan, China. JAMA 2020;323:1061-9.

2. Lu R, Zhao X, Li J, et al. Genomic characterisation and epidemiology of 2019 novel coronavirus: implications for 
virus origins and receptor binding. Lancet 2020;395:565-74.

3. Huang C, Wang Y, Li X, et al. Clinical features of patients infected with 2019 novel coronavirus in Wuhan, China. Lancet 2020;395:497-506.

4. Mahase E. Covid-19: WHO declares pandemic because of "alarming levels" of spread, severity, and inaction. BMJ 2020;368:m1036.

5. Cucinotta D, Vanelli M. WHO Declares COVID-19 a Pandemic. Acta Biomed 2020;91:157-60.

6. Chan JF, Yuan S, Kok KH, et al. A familial cluster of pneumonia associated with the 2019 novel coronavirus indicating person-to-person transmission: a study of a family cluster. Lancet 2020;395:514-23.

7. Lu H. Drug treatment options for the 2019-new coronavirus (2019-nCoV). Biosci Trends 2020;14:69-71.

8. Kadam RU, Wilson IA. Structural basis of influenza virus fusion inhibition by the antiviral drug Arbidol. Proc Natl Acad Sci U S A 2017;114:206-14.

9. Hulseberg CE, Fénéant L, Szymańska-de Wijs KM, et al. Arbidol and Other Low-Molecular-Weight Drugs That Inhibit Lassa and Ebola Viruses. J Virol 2019;93:e02185-18.

10. Wang X, Cao R, Zhang $\mathrm{H}$, et al. The anti-influenza virus drug, arbidol is an efficient inhibitor of SARS-CoV-2 in vitro. Cell Discov 2020;6:28.

11. Wang Z, Chen X, Lu Y, et al. Clinical characteristics and therapeutic procedure for four cases with 2019 novel coronavirus pneumonia receiving combined Chinese and Western medicine treatment. Biosci Trends 2020;14:64-8.

12. McKee DL, Sternberg A, Stange U, et al. Candidate drugs against SARS-CoV-2 and COVID-19. Pharmacol Res 2020;157:104859.

13. Nojomi M, Yassin Z, Keyvani H, et al. Effect of Arbidol (Umifenovir) on COVID-19: a randomized controlled trial. BMC Infect Dis 2020;20:954.

14. Lian N, Xie H, Lin S, et al. Umifenovir treatment is not associated with improved outcomes in patients with coronavirus disease 2019: a retrospective study. Clin Microbiol Infect 2020;26:917-21.

15. Lin L, Li TS. Interpretation of "Guidelines for the

Cite this article as: Chen N, Wang X, Zhang S, Lin R, Jiang Y. Efficacy analysis of Arbidol treatment in patients with 2019 novel coronavirus pneumonia: a retrospective cohort study. Ann Palliat Med 2021;10(10):10626-10632. doi: 10.21037/apm-212397
Diagnosis and Treatment of Novel Coronavirus (2019-nCoV) Infection by the National Health Commission (Trial Version 5)". Zhonghua Yi Xue Za Zhi 2020;100:805-7.

16. Phan LT, Nguyen TV, Luong QC, et al. Importation and Human-to-Human Transmission of a Novel Coronavirus in Vietnam. N Engl J Med 2020;382:872-4.

17. Jiang Y, He S, Zhang C, et al. Clinical characteristics of 60 discharged cases of 2019 novel coronavirusinfected pneumonia in Taizhou, China. Ann Transl Med 2020;8:547.

18. Wang M, Cao R, Zhang L, et al. Remdesivir and chloroquine effectively inhibit the recently emerged novel coronavirus $(2019-\mathrm{nCoV})$ in vitro. Cell Res 2020;30:269-71.

19. Choy KT, Wong AY, Kaewpreedee P, et al. Remdesivir, lopinavir, emetine, and homoharringtonine inhibit SARSCoV-2 replication in vitro. Antiviral Res 2020;178:104786.

20. Grein J, Ohmagari N, Shin D, et al. Compassionate Use of Remdesivir for Patients with Severe Covid-19. N Engl J Med 2020;382:2327-36.

21. Hazafa A, Ur-Rahman K, Haq IU, et al. The broadspectrum antiviral recommendations for drug discovery against COVID-19. Drug Metab Rev 2020;52:408-24.

22. Zumla A, Chan JF, Azhar EI, et al. Coronaviruses - drug discovery and therapeutic options. Nat Rev Drug Discov 2016;15:327-47.

23. Vankadari N. Arbidol: A potential antiviral drug for the treatment of SARS-CoV-2 by blocking trimerization of the spike glycoprotein. Int J Antimicrob Agents 2020;56:105998.

24. Deng L, Li C, Zeng Q, et al. Arbidol combined with LPV/ r versus LPV/r alone against Corona Virus Disease 2019: A retrospective cohort study. J Infect 2020;81:e1-5.

25. Yuan J, Zou R, Zeng L, et al. The correlation between viral clearance and biochemical outcomes of 94 COVID-19 infected discharged patients. Inflamm Res 2020;69:599-606.

(English Language Editor: J. Jones) 


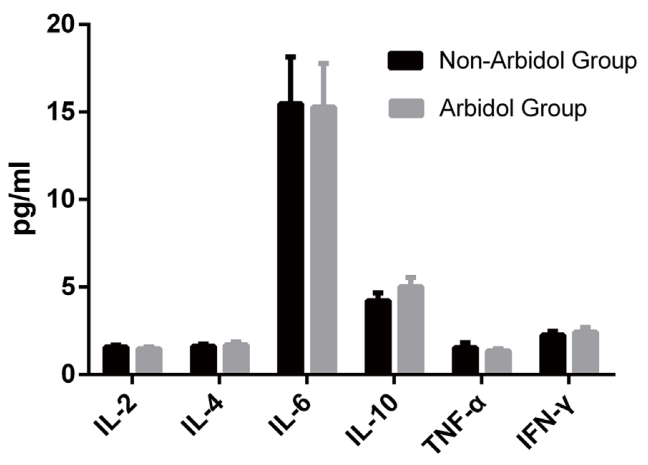

Figure S1 The levels of inflammatory factors, including IL-2, IL-4, IL-6, IL-10, TNF- $\alpha$, and IFN- $\gamma$, at the time of hospital admission. There were no differences between the Arbidol group and the non-Arbidol group. ARB, Arbidol; IL-2, interleukin-2; TNF- $\alpha$; tumor necrosis factor- $\alpha$; IFN- $\gamma$; interferon- $\gamma$. 
Table S1 Blood biochemistry and treatments

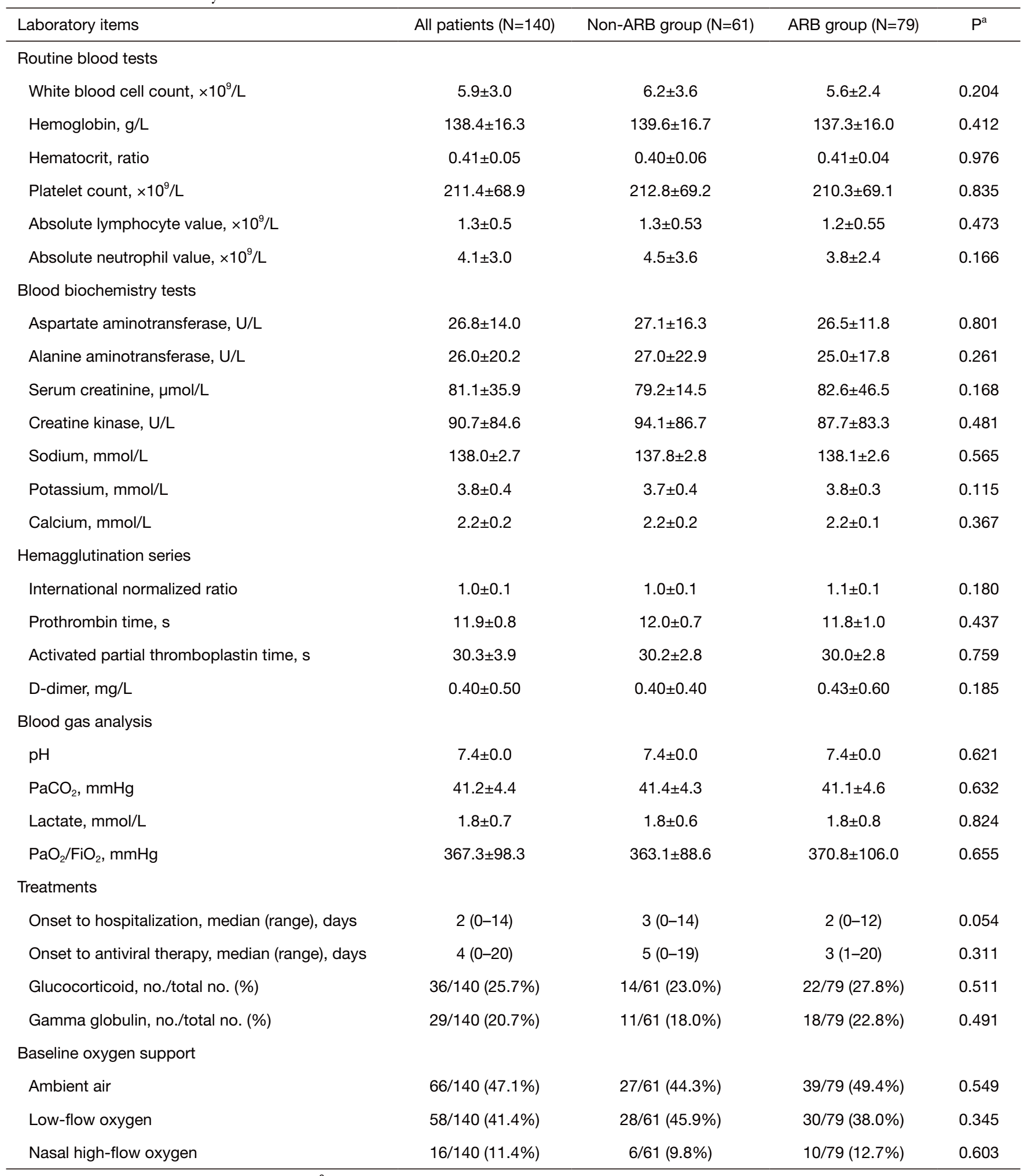

a, Student's $t$-test, Mann-Whitney $\mathrm{U}$ test, $\chi^{2}$ test and Fisher test. 